\title{
A radiometric normalization method of Controlling No-Changed Set (CNCS) for diverse landcover using multi-sensor data
}

\author{
LiTing Huang $^{1}$, WeiLi Jiao ${ }^{2, *}$, TengFei Long ${ }^{2}$, ChuanLi Kang ${ }^{1}$ \\ ${ }^{1}$ College of Geomatics and Geoinformation, Guilin University of Technology, Guilin, China \\ ${ }^{2}$ Institute of Remote Sensing and Digital Earth Chinese Academy of Sciences, Beijing, China
}

KEY WORDS: Multi-sensor, Radiometric Normalization, Automatic Scattergram-controlled Regression, Multivariate Alteration Detection, No-Changed Set

\begin{abstract}
:
The accurate acquisition of land surface reflectance (SR) data determines the accuracy of ground objects recognition, classification and land surface parameter inversion using remote sensing data, which is the basis of remote sensing data application. In this study, a Control No-Changed Set (CNCS) radiometric normalization method is proposed to realize spectral information transformation of multi-sensor data, which is based on the Iteratively Reweighted Multivariate Alteration Detection (IR-MAD), and includes automatic selection and step-by-step optimization of no-change pixels. The No-Changed set (NC) is obtained by selecting the original nochange pixels between the target image and the reference image according to the linear relationship. In the obtained original nochange regions, IR-MAD rules with iterative control are used to fix the final no-change pixels, after regression modeling and calculation, the normalized images are obtained. The method is tested on multi-images from multi-sensors in three groups of experiments (GF-1 WFV and Landsat-8 OLI, GF-1 PMS and Sentinel-2 MSI, and Landsat-8 OLI and Sentinel-2 MSI) with different landcover areas. The results of radiometric normalization are evaluated qualitatively and quantitatively. The data of the three groups of experiments have a high correlation (correlation coefficient $r$ values $>0.85$ ), indicating that they can be used together as complementary data. The Root Mean Squared Error (RMSE) values calculate from the NC between the reference and normalized target images are much smaller than those between the reference and original target images. The radiometric colour composition effects, and the typical ground objects spectral reflective curves of the reference and normalized target images are very similar after radiometric normalization. These results indicate that the CNCS method considers the linear relationship of the no-change pixels and is effective, stable, and can be used to improve the consistency of SR of multi-images from multi-sensors.
\end{abstract}

\section{INTRODUCTION}

The accurate acquisition of land surface reflectance (SR) data determines the accuracy of ground objects recognition (Zhang et al., 2015), land cover classification(Friedl et al., 2010) and land surface parameter inversion(Nazeer et al., 2017) using remote sensing data, which is the basis of remote sensing data application. At the same time, the variety of remote sensing satellites is increasing, which makes the remote sensing data resources multi-source. However, the response to the ground reflection spectrum of different satellite sensors is inconsistent, resulting in differences in SR acquired by different satellites. Therefore, it is necessary to improve the consistency between data by spectral radiometric normalization before multi-source data joint application. There are many methods about radiometric normalization at present: Dark Set-Bright Set Normalization (DB)(Hall et al., 1991), Pseudo-invariant Feature (PIF)(Schott et al., 1988), Automatic Scattergram-controlled Regression (ASCR)(Elvidge et al., 1995), and Iteratively Reweighted MAD (IR-MAD)(Canty and Nielsen, 2008), etc. Previous studies have evaluated the various radiometric normalization methods over different types and times of land covers images. For example, Lin et al. (2015) utilized principal component analysis to determine the major axis of the bitemporal image scatterplot and to extract PIFs with the determined major axis. In Zhong et al. (2016), a hierarchical regression method is proposed to reduce the radiometric differences for multitemporal images, which consists of extraction of the pseudo-invariant features (PIFs) and optimization of normalization parameters. Zhou et al. (2016) presented the utilization of normalized difference water index (NDWI) to select the original PIFs, and statistical rules with iterative control were used to fix the final PIFs. Recently, Syariz et al. (2019) proposed a constrained orthogonal regression, a common radiometric level located between bitemporal images is selected as the reference, which enforces pixel spectral signatures to be as consistent as possible during radiometric normalization while band regression quality is preserved. But these radiometric normalization methods do not involve the physical mechanism of remote sensing, and do not fully consider the influence factors such as atmosphere, so it is often impossible to obtain high-precision SR, which is not conducive to quantitative parameter inversion of multi-source remote sensing data.

In this study, a method is proposed to normalize radiometric using IR-MAD to Control No-Changed Set (CNCS). This normalization method is based on the regression modelling using invariant pixels known as No-Changed set (NC) from the target image and the reference image, which is a better approach than using global or half of the image pixels for radiometric normalization (Yang et al., 2000). To select the NC, the

* Corresponding author

E-mail address: jiaowl@radi.ac.cn (WeiLi Jiao) 
preliminarily no-change pixels are selected through scattergrams used in automatic scattergram-controlled regression, which is the conventional linear regression method. The final no-change pixels are acquired through the regularized IR-MAD rule on the preliminarily selected NC, then using orthogonal regression, and a normalized target image is obtained. The objectives of this study are:

1. Reduce the complexity of atmospheric correction. For the target image with unsatisfactory atmospheric correction effect, the SR can be improved by reference image with better accuracy.

2. Radiometric normalization is used to further improve the consistency of radiometric information from multi-sensors remote sensing images.

In this paper, GF-1 Wide Field of View (WFV) and Landsat-8 Operational Land Imager (OLI), GF-1 Panchromatic Multispectral Sensor (PMS) and Sentinel-2 Multi-Spectral Instrument (MSI), Landsat-8 OLI and Sentinel-2 MSI are taken as examples to carry out three groups of experiments.

The remainder of this paper is organized as follows: Section 2 describes the proposed method. Section 3 shows the experimental data. Section 4 presents the experimental results, and Section 5 provides the conclusions and future works.

\section{METHODOLOGY}

This study proposes a CNCS radiometric normalization method based on the Iteratively Reweighted Multivariate Alteration Detection (IR-MAD). This method does not require the image to have both dark and bright features, and can eliminate the influence of large sample noise on the radiometric correction results. The proposed method consists of four main steps, namely, preliminary extract $\mathrm{NC}$, final screening $\mathrm{NC}$, orthogonal regression, and radiometry transformation, which will be described in Sections 2.1, 2.2, and 2.3, respectively. Among them, Section 2.2 is the focus of this study.

\subsection{Preliminary Extract NC}

General relative radiometric normalization methods assume that radiometric relationships between the corresponding bands of target image and reference image are linear, and rectify the target image to a reference image through a linear transformation (Biday et al., 2010). The common form for linear radiometric normalization is given by

$$
y_{i}=a x_{i}+b
$$

where $y_{i}$ is the digital number (DN) of band $i$ in the reference image; $x_{i}$ is the DN of band $i$ in the target image; and a, b are normalization coefficients for band $i$.

First, the no-change pixels are selected from the reference image and the target image, and then the normalization coefficients are calculated, and finally radiometry transformation.

Elivdge et al. (1995) proposed the ASCR in relative radiometric normalization. The ASCR procedure locates the centers for land and water data clusters using the near-infrared (NIR) target image data versus reference image data scattergrams to establish an initial regression line, and then determine the NC. However, not all images have land and water conditions at the same time. Therefore, the CNCS differs from the ASCR method in that the former does not need the land and water data clusters on the scattergrams to determine the NC. It is on the scattergrams of the red and NIR bands, the orthogonal regression method (which is introduced in 2.3) is used to determine the initial regression baseline, and then further filters the no-change pixels to solve the NC. Solving the initial regression line is shown in Figure 1.

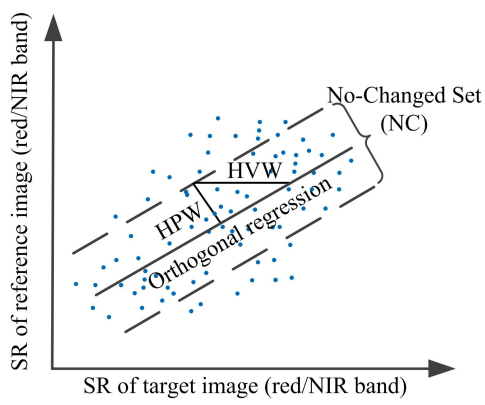

Figure 1. Determine the No-Change set (NC) from the scattergrams

HVW in the Fig. 2 is the restriction condition of NC, controlled by HPW, and HPW is the vertical width of one side of the NC. HPW, HVW and NC have the following relationship (Elvidge et al., 1995):

$$
\begin{gathered}
H V W_{N C}=\sqrt{1+a^{2}} * H P W_{N C} \\
N C=(X, Y):\left|y_{1}-b_{1}-a_{1} x_{1}\right| \leq H V W_{N C 1} \& \\
\left|y_{2}-b_{2}-a_{2} x_{2}\right| \leq H V W_{N C 2}
\end{gathered}
$$

Where $a$ is the slope of the initially estimated axis determined by orthogonal regression. Once the HVW is determined, the initial $\mathrm{NC}$ can be determined by Equation (3), where $\mathrm{y}_{1}$ and $\mathrm{y}_{2}$ are SR values of red band and NIR band of the reference image, respectively; $x_{1}$ and $x_{2}$ are SR values of red band and NIR band of the target image, respectively; and $a_{\mathrm{i}}$ and $\mathrm{b}_{\mathrm{i}}$ are coefficients determined by orthogonal regression.

The NC determined by the linear transformation roughly represents a "pseudo-invariant" pixel set, that is, a pixel whose radiation characteristics hardly change (Seo et al., 2017).

\subsection{Final screening $\mathrm{NC}$}

The quality of the no-change pixels affect the result of radiometric normalization, and further optimization of the nochange pixel is required in the NC. Use the following rules to find sample data that can be used for regression (Canty and Nielsen, 2008): Set the initial weight of each pixel as 1, calculate the mean vector and the variance matrix in each iteration, and calculate each MAD variate with the method of canonical correlation analysis; the weight of the pixels are updated according to the newly calculated MAD variates, and the weight range is $[0,1]$; the no-change pixel has a large weight, and after several iterations, the weight of each pixel tends to be stable; by comparing the weight with the threshold, it is possible to determine whether a pixel is a no-change pixel or not.

The formulas for updating weights are: 


$$
\begin{gathered}
\sigma_{M A D_{i}}^{2}=\gamma_{i}=2\left(1-\rho_{N-i+1}\right) \\
Z=\sum_{i=1}^{N} \frac{\left(M A D_{i}\right)^{2}}{\gamma_{i}} \in \chi^{2}(N) \\
P_{r}(\text { no change })=1-P_{\chi^{2} ; N}(Z)
\end{gathered}
$$

Where $\gamma_{i}$ is the variance of band $\mathrm{i}$, and $\mathrm{Z}$ is a random variate introduced by iterative assignment, which represents the sum of the standard MAD variates squares, obeys the chi-square distribution with degree of freedom $\mathrm{N}$ (band number), and is weighted by chi-square distribution probability density function. $P_{r}$ (no change) represents the iteration weight, and a fixed threshold $t$ can be set (for example, $t=0.95$ can be set to obtain a good result). When $P_{r}$ (no change $)>t$, the pixel can be assigned a label "no-change". This is described in detail in Canty and Nielsen (2008).

There are many (correlated) variables during the iteration, the solutions to the coupled generalized eigenvalue problems may become unstable due to (near) singular variance covariance matrices causing small changes in the data to lead to dramatically different solutions. Therefore, Nielsen(2007) added regularization (also known as penalization) in the iterative process to avoid the weight generated being too large, and affect the judgment of the no-change pixels. In this study, regularization parameter is also added when solving the covariance matrices:

$$
\begin{aligned}
& \operatorname{Var}_{11}=\sum_{11}+\lambda_{1} \Omega_{1} \\
& \operatorname{Var}_{22}=\sum_{22}+\lambda_{2} \Omega_{2}
\end{aligned}
$$

Where $\lambda_{i}$ is a regularization parameter, whose range is [0 1), and $\Omega_{\mathrm{i}}$ is a diagonal matrix of $\mathrm{N} \times \mathrm{N}$.

\section{3 orthogonal regression, and radiometry transformation}

Orthogonal regression method based on geometric distance is adopted (Leng et al., 1988). Suppose there are $\mathrm{k}$ data points $P_{i}\left(x_{i}, y_{i}\right), i=1 \cdots k$ in the two-dimensional plane, the regression analysis is to obtain a straight line $L$ as shown in formula (1) $y_{i}=a x_{i}+b$ by some criterion. Minimize $D=\sum_{i=1}^{k} d\left(P_{i}, L\right)$, where $d\left(P_{i}, L\right)$ is the geometric distance from point $P_{i}$ to $L$. In orthogonal regression, $a$ can be estimated according to

$$
\begin{gathered}
a=\frac{\left(S_{y y}^{2}-S_{x x}^{2}\right)+\sqrt{\left(S_{y y}^{2}-S_{x x}^{2}\right)^{2}+4 S_{x y}^{2}}}{2 S_{x y}} \\
S_{x x}^{2}=\frac{1}{n} \sum_{i=1}^{n}\left(x_{i}-\bar{x}^{2}\right. \\
S_{y y}^{2}=\frac{1}{n} \sum_{i=1}^{n}\left(y_{i}-\bar{y}^{2}\right. \\
S_{x y}=\frac{1}{n} \sum_{i=1}^{n}\left(x_{i}-\bar{x}\right)\left(y_{i}-\bar{y}\right)
\end{gathered}
$$

Where $\bar{x}$ and $\bar{y}$ is the mean of $\mathrm{x}, \mathrm{y}$, respectively. And $b$ can calculated according to $a$, that is $b=\bar{y}-a \bar{x}$.
After obtaining the $\mathrm{NC}$ in Section 2.2, the regression equation can be established in the $\mathrm{NC}$ for relative radiometric normalization from the above equations.

\section{DATA AND PRE-PROCESSING}

Sentinel-2 Level-2A are bottom of the atmosphere (BOA) reflectance products, which can be produced by Sen2cor, a processor for Level-2A data released by European Space Agency (ESA)(Louis et al., 2016). It is also available through the URL https://scihub.copernicus.eu/dhus/\#/home, and can be downloaded the Level-2A data in China region since December 2018.

Landsat-8 OLI Level-2 Surface Reflectance data are generated from the Land Surface Reflectance Code (LaSRC). LaSRC makes use of the coastal aerosol band to perform aerosol inversion tests, uses auxiliary climate data from MODIS and uses a unique radiative transfer model (Badawi et al., 2019). LaSRC is regarded as the most accurate for Landsat-8 OLI atmospheric correction, and Level-2 data can be freely downloaded at https://earthexplorer.usgs.gov/.

GF-1 has no relevant SR products released. The DN images are downloaded from China Center for Resource Satellite Data and Applications (http://www.cresda.com/CN/). DN images need to be pre-processed with atmospheric correction to obtain SR products. In this study, atmospheric correction of GF-1 images are performed based on ARCSI (Atmospheric and Radiometric Correction of Satellite Imagery) (Clewley et al., 2014).

As the resolution of the three satellites are inconsistent, resampling processing is needed before radiometric normalization to make the resolution of target image consistent

\begin{tabular}{|c|c|c|c|}
\hline Sensor & Spectral range $(\mu \mathrm{m})$ & $\begin{array}{l}\text { Resolution } \\
\text { (m) }\end{array}$ & $\begin{array}{c}\text { Revisit } \\
\text { periods(day) }\end{array}$ \\
\hline $\begin{array}{l}\text { GF-1 } \\
\text { WFV }\end{array}$ & $\begin{array}{ll}\text { Band1(blue): } & 0.45-0.52 \\
\text { Band2(green): } & 0.52-0.59 \\
\text { Band3(red): } & 0.63-0.69 \\
\text { Band4(NIR): } & 0.77-0.89\end{array}$ & 16 & 2 \\
\hline $\begin{array}{l}\text { GF-1 } \\
\text { PMS }\end{array}$ & $\begin{array}{ll}\text { Band1(blue): } & 0.45-0.52 \\
\text { Band2(green): } & 0.52-0.59 \\
\text { Band3(red): } & 0.63-0.69 \\
\text { Band4(NIR): } & 0.77-0.89\end{array}$ & 8 & $3 \sim 5$ \\
\hline $\begin{array}{l}\text { Landsat-8 } \\
\text { OLI }\end{array}$ & $\begin{array}{l}\text { Band2(blue): } \quad 0.45-0.51 \\
\text { Band3(green): } 0.53-0.59 \\
\text { Band4(red): } 0.64-0.67 \\
\text { Band5(NIR): } 0.85-0.88\end{array}$ & 30 & 16 \\
\hline $\begin{array}{l}\text { Sentinel-2 } \\
\text { MSI }\end{array}$ & $\begin{array}{ll}\text { Band2(blue): } & 0.46-0.52 \\
\text { Band3(green): } & 0.54-0.58 \\
\text { Band4(red): } & 0.65-0.68 \\
\text { Band8(NIR): } & 0.79-0.90\end{array}$ & 10 & 10 \\
\hline
\end{tabular}
with that of reference image.

In the experiments, three satellite images that contain various landscapes are used for relative radiometric normalization in four bands of blue, green, red and NIR, which verified the feasibility and performance of the proposed method. The properties of satellites are tabulated in Table 1 .

Table 1. Overview of the main parameters of the multiple sensors

The experiments are divided into three groups, Dataset 1 , Dataset 2 and Dataset 3, and all groups included three sets of experiments. Landsat-8 OLI is selected as the reference image in Dataset 1 and 3, and Dataset 2 selects the Sentinel-2 MSI as the reference image. The study area images should be selected on the same date as much as possible to ensure that the remote 
sensing images are less affected by the atmosphere. However, because different satellites have different revisit periods and swaths, it is difficult to obtain different satellite images of the same date and transit at the same time. In this study, the date difference between the target image and the reference image is no more than seven days. Three study areas, comprising various landscapes, including desert, agricultural crop, and urban area, are selected as shown in Table 2-4 for method evaluation.

Table 2. GF-1 WFV and Landsat-8 OLI study areas

\begin{tabular}{|c|c|c|c|}
\hline Dataset 1 & WFV4 OLI & WFV3 & WFV2 OL1 \\
\hline Path, row & 137,032 & 123,037 & 122,033 \\
\hline Acquisitions date & $\begin{array}{l}20180512, \\
20180512\end{array}$ & $\begin{array}{l}20180409, \\
20180408\end{array}$ & $\begin{array}{l}20180924, \\
20180924\end{array}$ \\
\hline Image size & $500 \times 500$ & $500 \times 500$ & $500 \times 500$ \\
\hline
\end{tabular}

Table 3. GF-1 PMS and Sentinel-2 MSI study areas

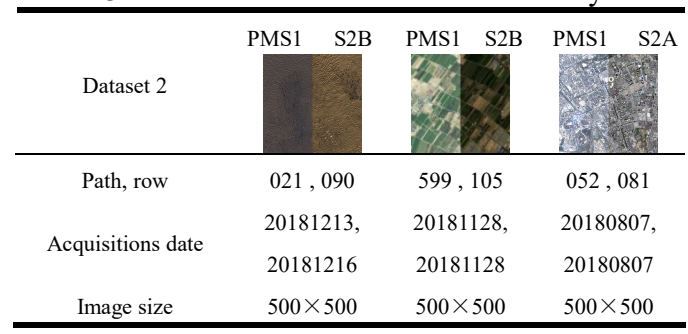

Table 4. Sentinel-2 MSI and Landsat-8 OLI study areas

\begin{tabular}{|c|c|c|c|}
\hline Dataset 3 & $\begin{array}{lll}\text { S2B } & \text { OLI }\end{array}$ & S2A OLI & $\begin{array}{ll}\text { S2B } & \text { OLI } \\
& \end{array}$ \\
\hline Path, row & 126,029 & 122,038 & 123,032 \\
\hline Acquisitions date & $\begin{array}{l}20181225, \\
20181230\end{array}$ & $\begin{array}{l}20180417, \\
20180420\end{array}$ & $\begin{array}{l}20181118 \\
20181121\end{array}$ \\
\hline Image size & $500 \times 500$ & $500 \times 500$ & $500 \times 500$ \\
\hline
\end{tabular}

\section{EXPERIMENTAL RESULTS AND DISCUSSION}

\subsection{The process of selecting NC}

Taking the reflectance of the target image band as the $\mathrm{X}$-axis and that of the reference image band as the Y-axis, the red and NIR band of the target image and the reference image respectively generate a scattergram to determine the initial regression line and extract NC. Taking Dataset 1 as an example, the NC scatterplots from step-by-step selection process for Dataset 1 are displayed in Figure 2. The black dots in the figure represent all the pixels of the image, the blue dots are the initially extracted $\mathrm{NC}$, the yellow dots are the last extracted $\mathrm{NC}$, the green lines represent the initial regression line, and the red lines represent the final fitted line. It should be noted that the SR values of the experimental images here are all scaled by 10,000 times.

These scattergrams have some common characteristics: The nochange pixels gradually converge, and the number of points in the last scatter diagram decreases, and are distributed along a straight line; the slope of the principal axis varies slightly from the second to the third time; the variation range of scattergrams gradually decrease, and all points are slightly evenly distributed.
These characteristics indicate the effectiveness and stability of the method in the selection of NC.

Table 5. Quantitative validation of desert study area

\begin{tabular}{cccccccc}
\hline \multirow{2}{*}{ Type } & \multirow{2}{*}{ Group } & \multirow{2}{*}{ Band } & Slope & Intercept & \multicolumn{3}{c}{ RMSE } \\
& & & $(a)$ & $(b)$ & Before & After & $r$ \\
\hline \multirow{4}{*}{ Dataset 1 } & blue & 0.496 & -582.591 & 2902.016 & 9.711 & 0.980 \\
& (WFV4 and & green & 0.515 & -259.834 & 2689.402 & 13.485 & 0.981 \\
& red & 0.558 & -374.429 & 2887.102 & 13.289 & 0.990 \\
& & NIR & 0.553 & -193.524 & 2843.463 & 14.231 & 0.992 \\
& Dl) & blue & 4.405 & -1562.813 & 437.6416 & 15.325 & 0.994 \\
Desert & Dataset 2 & green & 4.161 & -1792.187 & 898.047 & 20.603 & 0.995 \\
& (PMS1 and & red & 3.882 & -1917.920 & 1391.316 & 30.328 & 0.995 \\
& S2B) & NIR & 3.629 & -1672.024 & 1699.054 & 41.131 & 0.994 \\
& & blue & 0.691 & 421.698 & 133.165 & 11.753 & 0.976 \\
& Dataset 3 & green & 0.850 & 396.980 & 167.842 & 12.717 & 0.993 \\
(S2B and OLI) & red & 0.843 & 323.132 & 78.175 & 15.392 & 0.996 \\
& & NIR & 0.846 & 345.674 & 174.273 & 16.008 & 0.997 \\
\hline
\end{tabular}

Table 6. Quantitative validation of agricultural crop study area

\begin{tabular}{|c|c|c|c|c|c|c|c|}
\hline \multirow{2}{*}{ Type } & \multirow{2}{*}{ Group } & \multirow{2}{*}{ Band } & \multirow{2}{*}{$\begin{array}{l}\text { Slope } \\
(a)\end{array}$} & \multirow{2}{*}{$\begin{array}{c}\text { Intercept } \\
\text { (b) }\end{array}$} & \multicolumn{2}{|c|}{ RMSE } & \multirow[b]{2}{*}{$r$} \\
\hline & & & & & Before & After & \\
\hline \multirow{12}{*}{$\begin{array}{l}\text { Agricultura } \\
1 \text { crop }\end{array}$} & \multirow{4}{*}{$\begin{array}{c}\text { Dataset } 1 \\
\text { (WFV3 and } \\
\text { OLI) }\end{array}$} & blue & 1.765 & -843.482 & 356.120 & 20.992 & 0.857 \\
\hline & & green & 1.272 & -419.039 & 219.996 & 13.022 & 0.963 \\
\hline & & red & 1.177 & -209.879 & 141.956 & 11.596 & 0.972 \\
\hline & & NIR & 1.601 & -1723.864 & 624.098 & 42.485 & 0.989 \\
\hline & \multirow{4}{*}{$\begin{array}{c}\text { Dataset } 2 \\
\text { (PMS1 and } \\
\text { S2B) }\end{array}$} & blue & 0.676 & -667.739 & 1292.754 & 12.433 & 0.998 \\
\hline & & green & 0.683 & -511.636 & 1203.032 & 14.155 & 0.998 \\
\hline & & red & 0.719 & -564.539 & 1153.685 & 18.082 & 0.999 \\
\hline & & NIR & 0.754 & -893.043 & 2348.282 & 23.918 & 0.999 \\
\hline & \multirow{4}{*}{$\begin{array}{c}\text { Dataset } 3 \\
\text { (S2A and } \\
\text { OLI) }\end{array}$} & blue & 0.832 & -3.841 & 105.102 & 10.733 & 0.995 \\
\hline & & green & 0.732 & 195.700 & 56.784 & 11.026 & 0.995 \\
\hline & & red & 0.724 & 209.097 & 92.115 & 17.290 & 0.997 \\
\hline & & NIR & 0.683 & 856.039 & 309.409 & 66.33 & 0.990 \\
\hline
\end{tabular}

Table 7. Quantitative validation of urban area study area

\begin{tabular}{|c|c|c|c|c|c|c|c|}
\hline \multirow{2}{*}{ Type } & \multirow{2}{*}{ Group } & \multirow{2}{*}{ Band } & \multirow{2}{*}{$\begin{array}{c}\text { Slope } \\
(a)\end{array}$} & \multirow{2}{*}{$\begin{array}{c}\text { Intercept } \\
\text { (b) }\end{array}$} & \multicolumn{2}{|c|}{ RMSE } & \\
\hline & & & & & Before & After & \\
\hline \multirow{12}{*}{$\begin{array}{c}\text { Urban } \\
\text { area }\end{array}$} & \multirow{4}{*}{$\begin{array}{c}\text { Dataset } 1 \\
\text { (WFV2 and } \\
\text { OLI) }\end{array}$} & blue & 0.881 & -12.823 & 103.002 & 40.559 & 0.982 \\
\hline & & green & 0.985 & -74.5 & 90.613 & 36.662 & 0.989 \\
\hline & & red & 1.086 & -132.531 & 65.014 & 25.453 & 0.996 \\
\hline & & NIR & 1.374 & -665.786 & 242.263 & 35.079 & 0.998 \\
\hline & \multirow{5}{*}{$\begin{array}{l}\text { Dataset } 2 \\
\text { (PMS1 and } \\
\text { S2B) }\end{array}$} & blue & 0.789 & -988.000 & 1557.496 & 24.153 & 0.998 \\
\hline & & green & 0.705 & -585.814 & 1362.544 & 25.686 & 0.997 \\
\hline & & red & 0.707 & -508.159 & 1295.387 & 14.796 & 0.999 \\
\hline & & NIR & 0.740 & -763.092 & 1728.091 & 34.370 & 0.997 \\
\hline & & blue & 0.533 & 336.150 & 196.223 & 29.447 & 0.974 \\
\hline & \multirow{3}{*}{$\begin{array}{c}\text { Dataset } 3 \\
\text { (S2B and OLI) }\end{array}$} & green & 0.636 & 351.022 & 230.765 & 21.171 & 0.994 \\
\hline & & red & 0.669 & 327.021 & 221.335 & 20.915 & 0.996 \\
\hline & & NIR & 0.735 & 350.426 & 234.809 & 29.657 & 0.997 \\
\hline
\end{tabular}

\subsection{Qualitative and quantitative parameters statistical analysis}

To measure the overall differences, two-thirds of the sample points in the $\mathrm{NC}$ are regression fitted, and the rest are used as validation data to quantify the results of the radiometric normalization. Table 5-7 list the normalization coefficients for each band for the three group experiments. All of the experiments are quantitatively evaluated using the root-meansquare error (RMSE) and correlation coefficient $(r)$. RMSE is defined as $R M S E=\sqrt{1 / N \sum_{i=1}^{N}\left(y_{k}^{\prime}-y_{k}\right)^{2}}$, where $y_{k}^{\prime}$ is the SR value of target image band $k$ after radiometric normalization, $y_{k}$ is the SR value of reference image band $\mathrm{k}$, and $N$ is the number of samples. And $r$ is defined as 


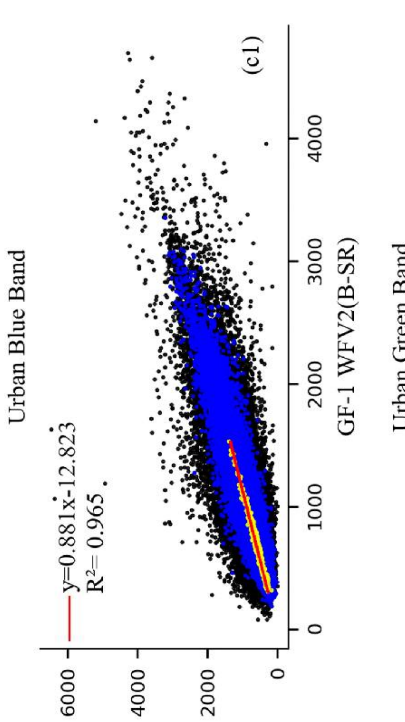

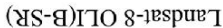

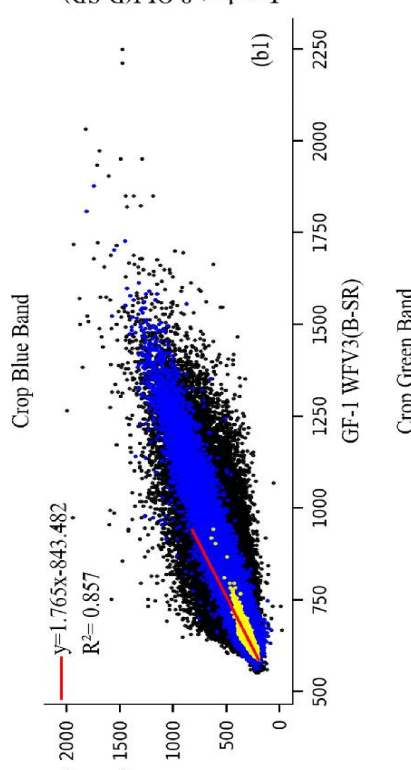

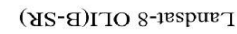

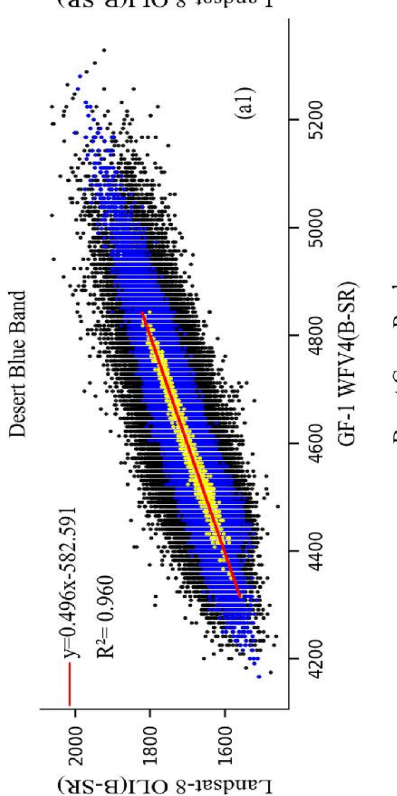

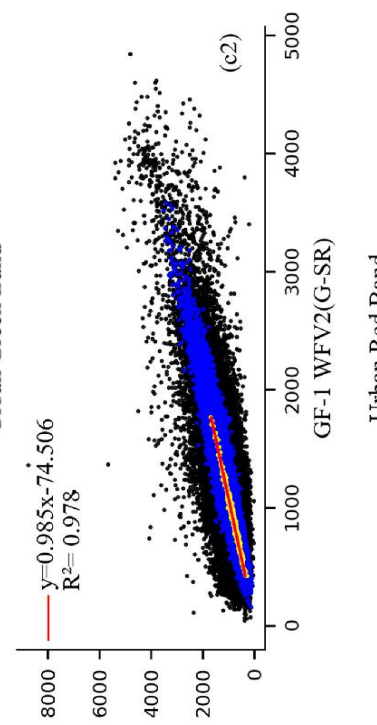

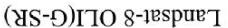
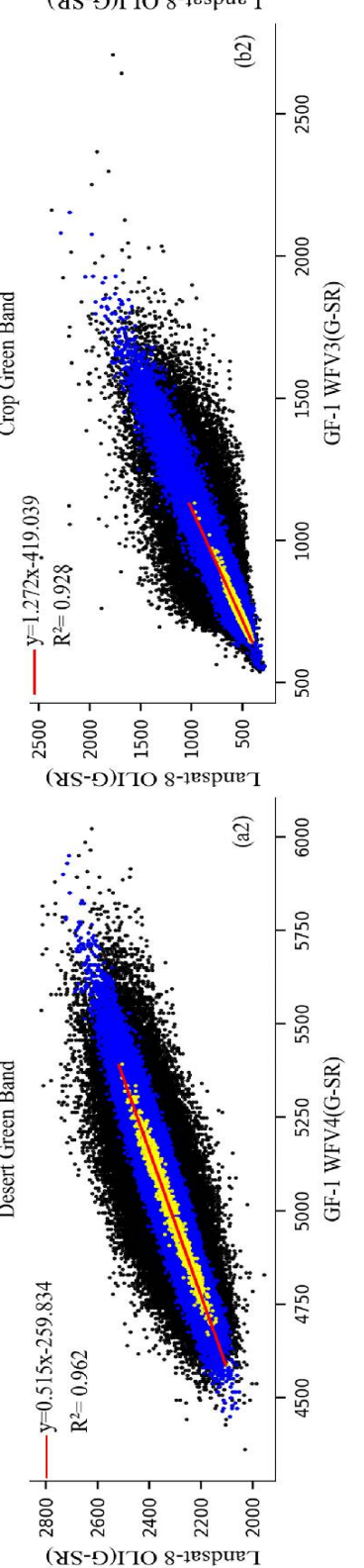

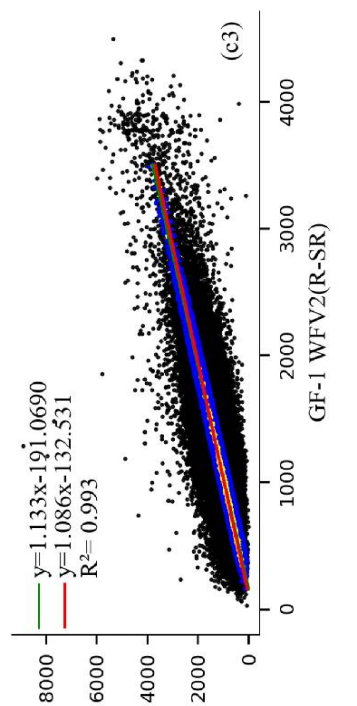

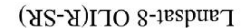
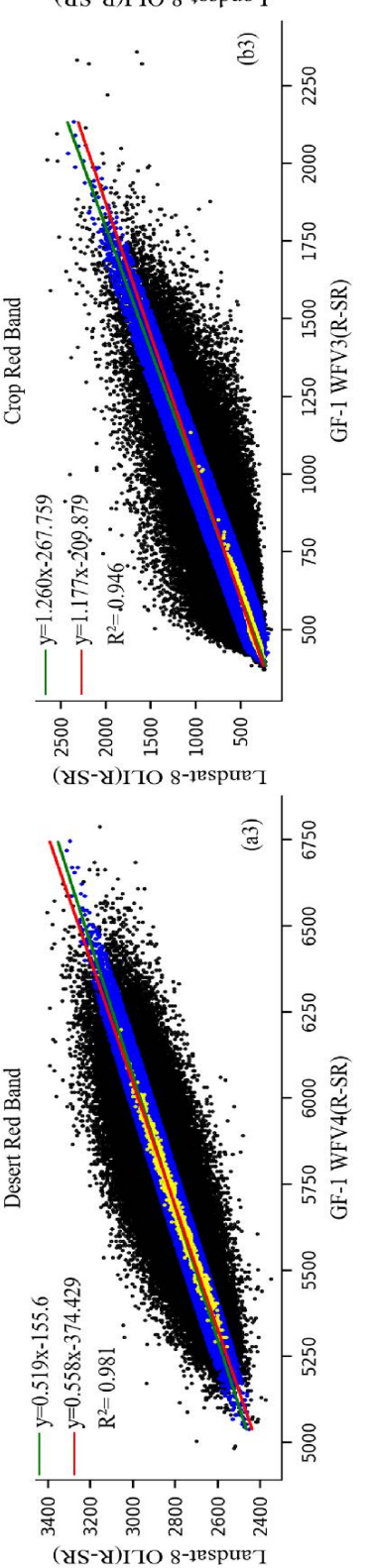
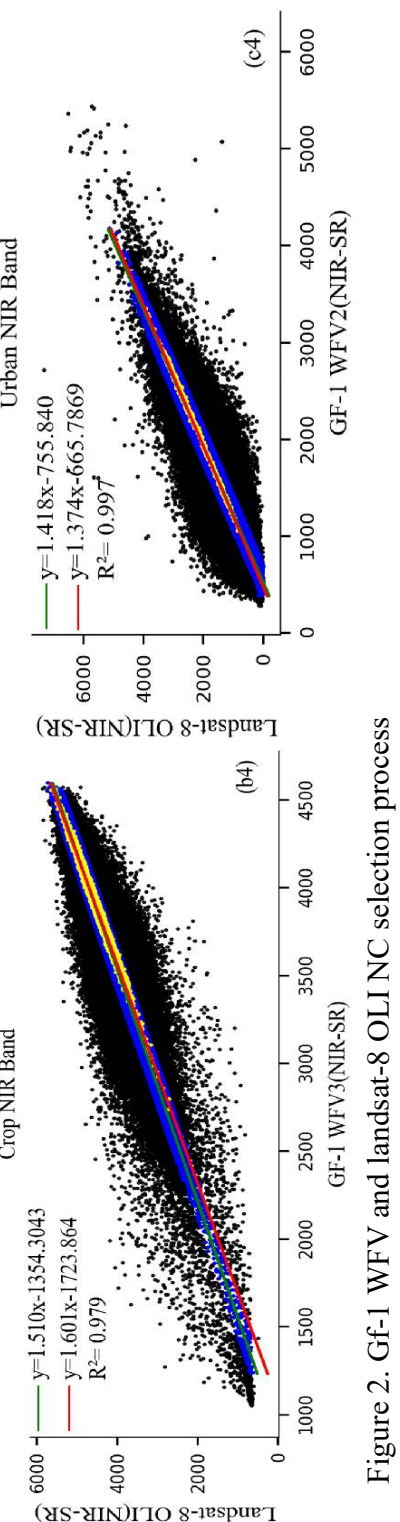

(УS-YIN)ITO 8-१espur]

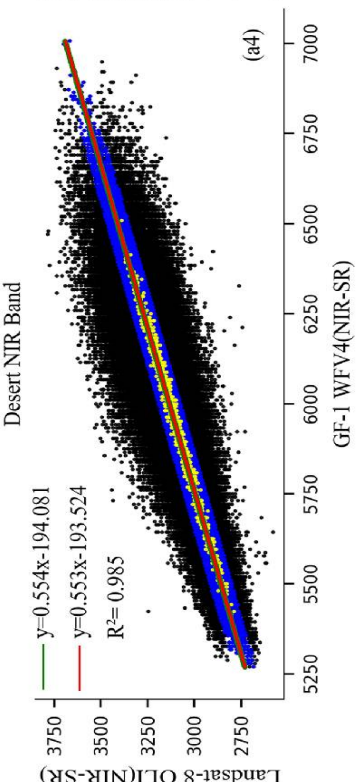


The International Archives of the Photogrammetry, Remote Sensing and Spatial Information Sciences, Volume XLII-3/W10, 2020 International Conference on Geomatics in the Big Data Era (ICGBD), 15-17 November 2019, Guilin, Guangxi, China
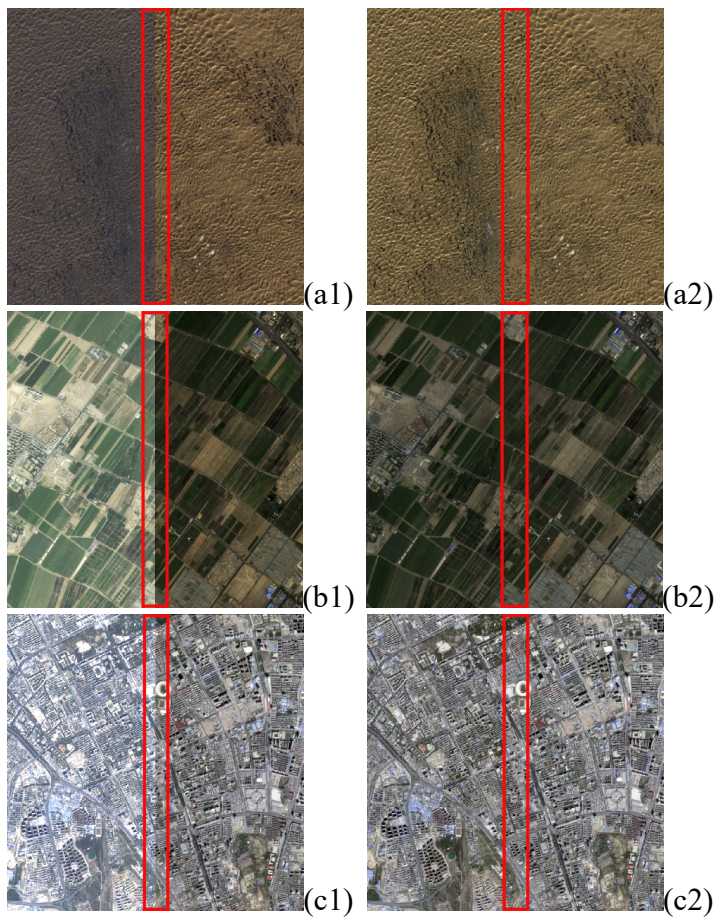

(a2)

(b2)
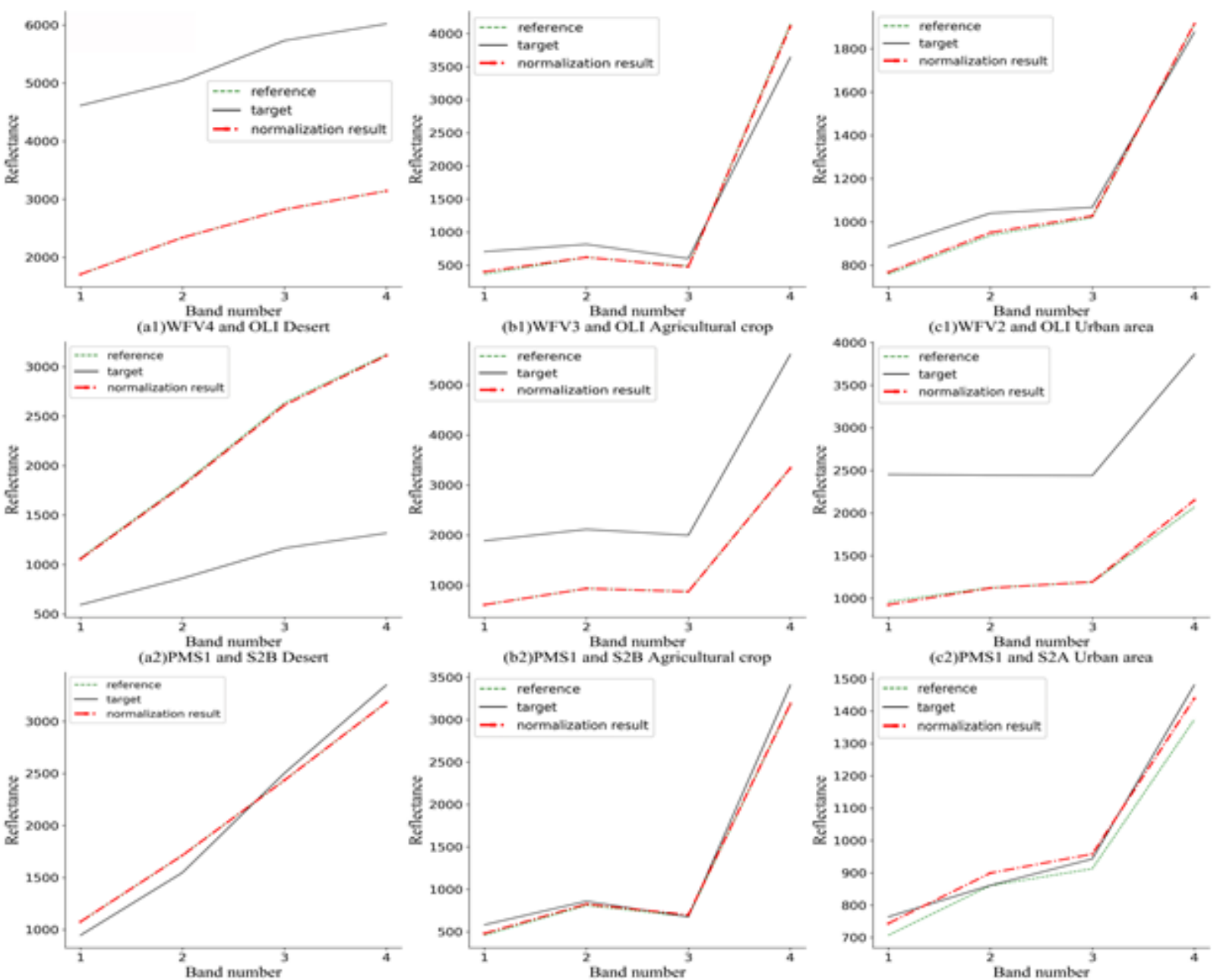

(a3)\$2 B and OLIDesen

(b3) $\mathrm{S} 2 \mathrm{~A}$ and $\mathrm{OLL} 1 \mathrm{Ag}$ ricultural crop

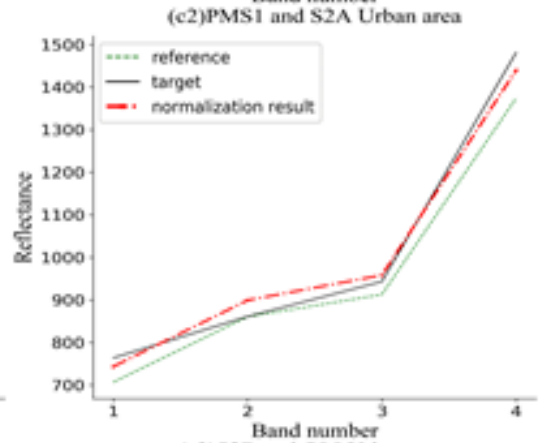

Figure 4. Comparison of spectral reflective curves of the ground objects before and after normalization 
$r\left(y_{k}^{\prime}, y_{k}\right)={\operatorname{Cov}\left(y_{k}^{\prime}, y_{k}\right)}_{\sqrt{\operatorname{Var}\left[y_{k}^{\prime}\right] \operatorname{Var}\left[y_{k}\right]}}$. From Table 5-7, it can be seen that the RMSE of the normalized image is smaller than that before radiometric normalization, and the $r$ values of the bands exceed 0.857 , and the highest $r$ is 0.999 , which shows that CNCS method can effectively reduce the radiometric differences between the target image and the reference image, and the correlation between the target and reference data is closer after radiometric normalization.

In addition, from the normalization coefficients, we can see the difference of SR products of different satellite sensors. In the desert area, the slope coefficients of GF-1 WFV and Landsat-8 are much less than 1, and the slope coefficients of GF-1 PMS and Sentinel-2B are much greater than 1, indicating that the ARCSI atmospheric correction method is not effective enough in desert area. This is due to the ARCSI method uses the dark dense vegetation approach to retrieve aerosols for atmospheric correction, while there is a lack of sufficient vegetation in the desert area.

The result of radiometric normalization is qualitatively evaluated by visual comparison. The normalized image is compared with the reference image, if the color and brightness of the two images are very similar, the normalization effect is better. Only the visual comparison results of Dataset 2 are listed here. The image colour composition effects of the normalized target images are more similar to those of the reference images than to those of the original target images. The radiometric differences between the original target and reference images are greatly reduced. These results are observed for all landcover areas from Figure 3.

Due to the radiometric distortion and the characteristics of different sensors, the spectral reflective curve characteristics of the ground objects are very different. One of the purposes of CNCS is to eliminate or reduce these differences. The more similar the spectral reflective curves of the ground objects are, the closer the spectral characteristics of the ground objects are, and the variation of the spectral curve can indicate the effect of relative radiometric normalization to a certain extent. It can be seen from Figure 4 that the spectral reflective curve of the radiometric normalized image is closer to the reference image, indicating that the radiometric normalization is effective.

It can be seen from the above results that although the atmospheric correction effects of different satellite images are not the same, the CNCS relative radiometric normalization method can improve the SR accuracy of the target image based on the reference image with better atmospheric correction effect. The purpose of reducing the complexity of atmospheric correction and improving the radiation consistency of multisource images is achieved.

\section{CONCLUSIONS}

A Control No-Changed Set (CNCS) radiometric normalization method is proposed. To validate the method under as many different conditions as possible, three groups of experiments with different landscape types are carried out. The results show that the NC selection method is consistently effective. The results of the three groups of experiments using the CNCS method are that: (1) The RMSE values calculated from the NC between the reference and normalized target images are much smaller than those between the reference and original target images, and the $r$ values of each band are close to 1 ; (2)
Visually comparing the results, the differences in the image edge of different landscapes (desert, agricultural crop, and urban area) are significantly reduced, the colour composition effects of the images after radiometric normalization are very similar; (3) After radiometric normalization, the spectral reflective curves of the typical ground objects are close to the reference images, indicating that the radiometric normalization is effective.

The uncertainties in the results are primarily caused by two factors. First, the uncertainties may have been caused by image registration errors. The geometrical registration errors are nearly one or two pixels, which would cause a mismatch of the nochange pixels locations between the original target and reference images. Second, the spectral range differences and spectral response differences among multiple sensors will affect the result of atmospheric correction, resulting in differences of reflectivity of the same ground object on different images. In near future, the spectral range differences and spectral response differences among multi-sensor will be studied.

\section{ACKNOWLEDGEMENTS}

This work has been supported by the Strategic Priority Research Program of the Chinese Academy of Sciences (grant number XDA19090300), the National Natural Science Foundation of China (grant number 61731022), the National Key R\&D Program of China (grant number 2016YFA0600302).

\section{REFERENCES}

Badawi, M., Helder D., Leigh, L., Jing, X., 2019. Methods for Earth-Observing Satellite Surface Reflectance Validation. Remote Sens. 11(13): 1543.

Biday, S.,G., Bhosle, U., 2010. Radiometric correction of multitemporal satellite imagery. Journal of Computer Science. 6(9): 940-949.

Canty, M.J., Nielsen, A.A., 2008. Automatic radiometric normalization of multitemporal satellite imagery with the iteratively re-weighted MAD transformation. Remote Sens. Environ. 112(3): 1025-1036.

Clewley, D., Bunting, P., Shepherd, J., Gillingham, S., Flood, N., Dymond, J., Lucas, R., Armston, J., Moghaddam, M., 2014. A Python-Based Open Source System for Geographic ObjectBased Image Analysis (GEOBIA) Utilizing Raster Attribute Tables. Remote Sensing. 6: 6111-6135.

Elvidge, C.D., Yuan, D., Weerackoon, R.D., Lunetta, R.S., 1995. Relative radiometric normalization of landsat Multispectral Scanner (MSS) data using an automatic scattergram-controlled regression. Photogramm. Eng. Remote Sens. 61(10): 1255-1260.

Friedl, M.A., Sulla-Menashe, D., Tan, B., Schneider, A., Ramankutty, N., Sibley, A., Huang, X., 2010. MODIS Collection 5 global land cover: Algorithm refinements and characterization of new datasets. Remote Sens. Environ. 114, 168-182.

Hall, F.G., Strebel, D.E., Nickeson, J.E., Goetz, S.J., 1991. Radiometric rectification: Toward a common radiometric response among multidate, multisensor images. Remote Sens. Environ. 35(1), 11-27. 
Leng, L., Zhang, T.Y., Kleinman, L., Zhu, W., 2007. Ordinary Least Square Regression, Orthogonal Regression, Geometric Mean Regression and their Applications in Aerosol Science. Journal of Physics Conference Series. 78(1): 1-5.

Lin, C.H., Lin, B.Y., Lee, K.Y., Chen, Y.C., 2015. Radiometric normalization and cloud detection of optical satellite images using invariant pixels. ISPRS J. Photogramm. Remote Sens. $106,107-117$.

Louis, J., Debaecker, V., Pflug, B., Main-Knorn, M., Bieniarz, J., Müller-Wilm, U., Cadau, E., Gascon, F., 2016. Sentinel-2 Sen2cor: L2A processor for users. ESA Living Planet Symposium. 6(9): 1027-1036.

Nazeer, M.; Wong, M.S.; Nichol, J.E., 2017. A new approach for the estimation of phytoplankton cell counts associated with algal blooms. Sci. Total Environ. 590, 125-138.

Nielsen, A.A., 2007. The regularized iteratively reweighted MAD method for change detection in multi- and hyperspectral data. IEEE Transactions on Image Processing. 16(2): 463-478.

Schott, J.R., Salvaggio, C., Volchok W J. 1988. Radiometric scene normalization using pseudoinvariant features. Remote Sens. Environ. 26(1): 1-16.

Seo, D.K., Yong, H.K., Yang, D.E., Wan, Y.P., 2017. Generation of radiometric, phenological normalized image based on random forest regression for change detection. Remote Sensing. 9(11): 1-21.

Syariz, M.A., Lin, B.Y., Denaro L.G., Jaelani, L.M., Nguyen, M.V., Lin, C.H., 2019. Spectral-consistent relative radiometric normalization for multitemporal Landsat 8 imagery. Int. J. Remote Sens.147:56-64.

Yang, X.J., Lo, C.P., 2000. Relative radiometric normalization performance for change detection from multi-date satellite images. Photogramm. Eng. Remote Sens. 66(8): 967-980.

Zhang, R., Qu, J.J., Liu, Y., Hao, X., Huang, C., Zhan, X., 2015. Detection of burned areas from mega-fires using daily and historical MODIS surface reflectance. Int. J. Remote Sens. 36, 1167-1187.

Zhong, C., Xu, Q., Li, B., 2016. Relative radiometric normalization for multitemporal remote sensing images by hierarchical regression. IEEE Geosci. Remote Sens. Lett. 13 (2), 217-221.

Zhou, H., Liu, S., He, J., Wen, Q., Song, L., Ma, Y., 2016. A new model for the automatic relative radiometric normalization of multiple images with pseudo-invariant features. Int. J. Remote Sens. 37 (19), 4554-4573. 